\title{
Transferrin-Associated Lipoplexes as Gene Delivery Systems: Relevance of Mode of Preparation and Biophysical Properties
}

\author{
Nuno Penacho $\cdot$ Ana Filipe · Sérgio Simões • \\ Maria C. Pedroso de Lima
}

Received: 16 October 2007/Accepted: 7 January 2008/Published online: 21 February 2008

(C) Springer Science+Business Media, LLC 2008

\begin{abstract}
The successful application of gene therapy depends highly on understanding the properties of gene carriers and their correlation with the ability to mediate transfection. An important parameter that has been described to improve transfection mediated by cationic liposomes involves association of ligands to cationic liposome-DNA complexes (lipoplexes). In this study, ternary complexes composed of 1,2-dioleoyl-3-(trimethylammonium) propane:cholesterol, plasmid DNA and transferrin (Tf, selected as a paradigm of a ligand) were prepared under various conditions, namely, in medium with different ionic strengths (HEPES-buffered saline [HBS] or dextrose), at different lipid/DNA (+/-) charge ratios and using different modes for component addition. We investigated the effect of these formulation parameters on transfection (in the absence and presence of serum), size of the complexes, degree of DNA protection and extent of their association with cells (in terms of both lipid and DNA). Our results show that all the tested parameters influenced to some extent the size of the complexes and their capacity to protect the carried genetic material, as well as the levels of cell association and transfection. The best transfection profile was observed for ternary complexes (Tf-complexes)
\end{abstract}

N. Penacho - A. Filipe · S. Simões - M. C. Pedroso de Lima ( $\square)$

Center for Neuroscience and Cell Biology,

University of Coimbra, Coimbra 3000, Portugal

e-mail: mdelima@ci.uc.pt

\section{A. Filipe $\cdot$ S. Simões}

Laboratory of Pharmaceutical Technology, Faculty of Pharmacy,

University of Coimbra, Coimbra 3000, Portugal

N. Penacho $\cdot$ M. C. Pedroso de Lima

Department of Biochemistry, Faculty of Science

and Technology, University of Coimbra, Coimbra 3000, Portugal prepared in high ionic strength solution (HBS), at charge ratios close to neutrality and according to the following order of component addition: cationic liposomes-Tf-DNA. Interestingly, in contrast to what was found for dextrose-Tfcomplexes, transfection mediated by HBS-Tf-complexes in the presence of serum was highly enhanced.

Keywords Cationic liposome - Transfection . Gene delivery $\cdot$ Biophysical property $\cdot$ Ternary complex . Serum

\section{Introduction}

Gene therapy is viewed as a promising strategy for the treatment of diseases with complex etiologies, such as cancer (Galanis \& Russell, 2001), neurodegenerative diseases (Baekelandt et al., 2000) and AIDS (Dropulic \& June, 2006), or to prevent allergic response (Horner et al., 2001). Gene therapy involves essentially two main entities, the therapeutic gene and a vector responsible for its transport to the target spot, ensuring that it arrives intact. Although viral vectors exhibit capacity to efficiently mediate gene delivery, they present several disadvantages including immunogenicity and oncogene activation (Mulligan, 1993). Therefore, nonviral vectors appear to be a viable alternative to viral gene delivery vehicles since they can obviate such limitations. Nevertheless, this kind of vector does not exhibit the transfection efficiency achieved by viral vectors; thus, the drive to improve nonviral vectors continues. Among nonviral vectors, cationic liposomes have been the most extensively studied due to their capacity to efficiently condense and protect DNA. The use of cationic liposome-DNA complexes (lipoplexes) to mediate transfection both in vitro (Lewis et al., 1996; 
Solodin et al., 1995; Vitiello et al., 1998) and in vivo (da Cruz et al., 2005; Liu et al., 1997; Mahato et al., 1998; Shirasaka et al., 1991; Wheeler et al., 1996) has been reported. However, gene delivery mediated by plain lipoplexes is not yet considered satisfactory, particularly when compared to viral vectors; and several strategies have been applied to increase their transfection capacity. In this regard, association of ligands to lipoplexes has been shown to be a promising approach to enhance their capacity to mediate gene delivery (da Cruz et al., 2001; Harvie, Wong $\&$ Bally, 2000). This strategy has been shown to increase complex-cell interaction and cellular internalization and to facilitate intracellular trafficking (da Cruz et al., 2001). Examples of proteins or peptides that upon association to cationic liposomes lead to enhancement of transfection include folate (Lee \& Huang, 1996), transferring (Tf) (da Cruz et al., 2001), insulin (Huckett, Ariatti \& Hawtrey, 1990), epidermal growth factor (EGF) (Kikuchi et al., 1996) and integrin-binding motifs (Harvie et al., 2000).

The present work involves a systematic study aimed at gaining insight into the experimental parameters that modulate both physicochemical and biological properties of ternary complexes composed of DNA, cationic liposomes and a protein (ligand).

In our laboratory, association of the ligand $\mathrm{Tf}$, a blood protein responsible for the transport of iron into cells, to lipoplexes has been extensively studied. We have shown that such association results in a significant enhancement of transfection in a large variety of cell types (da Cruz et al., 2001, 2005; da Cruz, Simoes \& de Lima, 2004; Neves et al., 2006; Simoes et al., 1999); therefore, in the present work, Tf was selected as a model protein.

The biophysical properties of cationic liposome-DNA complexes have been shown to be very important in dictating their mode of interaction with cells and ability to mediate gene delivery. In this study, we aimed at understanding how the different modes of preparation of ternary complexes composed of cationic lipid, plasmid DNA and a ligand (e.g., Tf) affect their physicochemical properties and consequently influence their biological activity. For this purpose, Tf-associated complexes (prepared under different experimental conditions) were studied in terms of their physicochemical properties and capacity to promote transfection in cultured COS-7 cells. The examined parameters were ionic strength of the medium where the complexes were prepared (high ionic strength, HEPESbuffered saline solution; low ionic strength, 5\% dextrose [Dex] solution), cationic liposome/DNA charge ratio $(3 / 2$, $2 / 1$ and 4/1) and mode of preparation (order 1 , cationic liposomes + Tf + pDNA; order 2, cationic liposomes + pDNA + Tf). The effect of the presence of serum on transfection mediated by the described complexes was also investigated. The mode of complex preparation drastically influenced the physicochemical properties of the ternary complexes, which in turn play a determinant role in their transfection ability. Moreover, our observations of the effect of serum on transfection mediated by Tf-complexes can be of great importance when planning the in vivo application of this kind of system.

\section{Materials and Methods}

Cationic Liposomes and Their Complexes with DNA and $\mathrm{Tf}$

Small unilamellar vesicles (SUVs) were prepared from a 1:1 (molar ratio) mixture of 1,2-dioleoyl-3-(trimethylammonium) propane (DOTAP) and cholesterol (Chol) by extrusion of multilamellar vesicles (MLVs). Briefly, DOTAP (Avanti Polar Lipids, Alabaster, AL) and Chol (Sigma, St. Louis, MO) dissolved in $\mathrm{CHCl}_{3}$ were mixed at a 1:1 molar ratio and dried under vacuum in a rotatory evaporator. The dried lipid films were hydrated with deionized water to a final lipid concentration of $6 \mathrm{~mm}$, and the resulting MLVs were then sonicated (in an ultrasonic bath) for $5 \mathrm{~min}$ and extruded 21 times, through two stacked polycarbonate filters of $50 \mathrm{~nm}$ pore diameter using a Lipofast device (Avestin, Toronto, Canada). The resulting liposomes (SUVs) were then diluted five times with deionized water and filter-sterilized utilizing $0.22 \mu \mathrm{m}$ porediameter filters (Schleicher \& Schuell, BioScience, Germany). Ternary complexes (Tf-complexes) were prepared by sequentially mixing $100 \mu \mathrm{l}$ of a HEPES-buffered saline solution (HBS; $100 \mathrm{~mm} \mathrm{NaCl,} 20 \mathrm{~mm}$ HEPES, pH 7.4) or $100 \mu \mathrm{l}$ of $5 \%$ (w/v) Dex, solution, $\mathrm{pH} 7.4$, with liposomes (volume was dependent on the desired [+/-] lipid/DNA charge ratio) and with $32 \mu \mathrm{l}$ of HBS or Dex solution containing $32 \mu \mathrm{g}$ of holo-Tf (Sigma). The mixture was incubated for $15 \mathrm{~min}$ at room temperature. Then, $68 \mu \mathrm{l}$ of HBS or Dex solution containing $1 \mu \mathrm{g}$ of pCMVluc (VR1216; a gift of Dr. P Felgner, Vical, San Diego, CA) were added to the previous solution and further incubated for 15 min. This mode of complex preparation was designated as "order 1." Alternatively, ternary complexes were prepared by changing the order of addition of $\mathrm{Tf}$ and pCMVluc (order 2).

Cells

COS-7 cells (American Type Culture Collection, Rockville, MD) were maintained at $37^{\circ} \mathrm{C}$ under $5 \% \quad \mathrm{CO}_{2}$ in Dulbecco's modified Eagle's medium-high glucose (DMEM-HG) (Irvine Scientific, Santa Ana, CA) supplemented with $10 \%(\mathrm{v} / \mathrm{v})$ heat-inactivated fetal bovine serum (FBS, Sigma), penicillin (100 U/ml), streptomycin 
$(100 \mu \mathrm{g} / \mathrm{ml})$ and L-glutamine (4 mM). For transfection, 45 $\times 10^{5} \mathrm{COS}-7$ cells were seeded in $1 \mathrm{ml}$ of medium in 48well culture plates and used at $50-70 \%$ confluence. Cell viability was determined by Trypan blue exclusion.

\section{Transfection Activity}

COS-7 cells were rinsed twice with serum-free medium and then covered with $0.3 \mathrm{ml}$ of DMEM-HG (without serum) before Tf-complexes were added. Tf-complexes containing $1 \mu \mathrm{g}$ of plasmid were added gently to the cells in a volume of $0.2 \mathrm{ml} /$ well. In experiments performed in the presence of serum, serum was added to the medium at different concentrations and maintained during the transfection process. After 4-h incubation (in $5 \% \mathrm{CO}_{2}$ at $37^{\circ} \mathrm{C}$ ), the medium was replaced with DMEM-HG containing $10 \% \mathrm{FBS}$ and the cells were further incubated for $48 \mathrm{~h}$. The cells were then washed twice with phosphate-buffered saline (PBS), and $100 \mu \mathrm{l}$ of lysis buffer (1 mm dithiothreitol, $1 \mathrm{~mm}$ EDTA, $25 \mathrm{~mm}$ Trisphosphate [pH 7.8], $8 \mathrm{~mm} \mathrm{MgCl}_{2}, 15 \%$ glycerol, $1 \%$ [v/v] Triton X-100) were added to each well. The level of gene expression in the lysates was evaluated by measuring light production by luciferase in a Mediators $\mathrm{PhL}$ luminometer (Mediators Diagnostika, Vienna, Austria). The protein content of the lysates was measured by the Sedmak method (Sedmak \& Grossberg, 1977).

\section{Mean Diameter of Tf-Complexes}

Complexes were characterized with respect to their size by photon correlation spectroscopy (PCS) using a Coulter N4 Plus (Coulter, Miami, FL). The PCS technique uses autocorrelation spectroscopy of scattered laser light to determine its time-dependent fluctuations resulting from the brownian motion of particles in suspension. The light intensity scattered at a given angle is detected by a photomultiplier, whose output current is passed to an autocorrelator, which analyzes time dependence, determining the rate of diffusion or brownian motion of the particles and, hence, their size. The detection angle is fixed at $90^{\circ}$. Tf-complexes were prepared immediately before analysis.

\section{PicoGreen Accessibility Assay}

Tf-complexes containing $1 \mu \mathrm{g}$ of DNA were incubated with inactive or active DNase for $30 \mathrm{~min}$ at $37^{\circ} \mathrm{C}$, using 10 units of DNase I per microgram of DNA. The accessibility of PicoGreen $^{\circledR}$ (Molecular Probes, Wageningen, the Netherlands) to the DNA associated with the complexes was monitored for $2 \mathrm{~min}$ in a SPEX Fluorolog 2 fluorometer (SPEX Industries, Edison, NJ). Fluorescence was read at excitation and emission wavelengths of 480 and $520 \mathrm{~nm}$, respectively, using 1.25 and $2.5 \mathrm{~mm}$ emission slits. The sample chamber was equipped with a magnetic stirring device. The experiments were performed at room temperature.

\section{Cell Association}

Cell association experiments were performed at $37^{\circ} \mathrm{C}$ under the same experimental conditions described for transfection. COS-7 cells were incubated in a final volume of $1 \mathrm{ml}$ (serum-free DMEM-HG) with Tf-complexes containing cationic lipid labeled with $5 \mathrm{~mol} \%$ of the fluorescent probe rhodamine-phosphoethanolamine (RhPE) (Avanti Polar Lipids, Alabaster, AL). After incubation, the medium containing the nonassociated Tf-complexes was collected and diluted to a final volume of $2 \mathrm{ml}$ of serum-free DMEM-HG. Fluorescence was measured at $37^{\circ} \mathrm{C}$ following addition of Triton X-100 (Merck, Darmstadt, Germany) at a final concentration of $0.5 \%(\mathrm{v} / \mathrm{v})$. To assess the fluorescence associated with the cells, cells were rinsed with serum-free DMEM-HG, detached from the culture dishes and then suspended in $2 \mathrm{ml}$ of medium. The fluorescence of the cell suspension was measured in the presence of Triton X-100 as described above. The extent of cell association (in terms of lipid) was determined according to the following equation:

$\%$ cell association $=\left[F_{\text {cells }} /\left(F_{\text {nonassociated }}+F_{\text {cells }}\right)\right] \times 100$

where $F_{\text {cells }}$ is the value of fluorescence associated with the cells and $F_{\text {nonassociated }}$ is the value of fluorescence of nonassociated Tf-complexes.

Alternatively, nucleotides labeled with radioactive ${ }^{35} \mathrm{~S}$ were diluted in the pDNA solution before complexation with the cationic liposomes and addition of $\mathrm{Tf}$ to form the final Tf-complexes. pDNA labeling was performed as recommended by the instructions of the Random Primed DNA Labeling Kit (Roche, Lewes, UK). The extent of association of the complexes with the cells in terms of DNA was determined using the experimental procedure described above, the cell suspension and medium being collected for subsequent radioactive quantification (which was performed in a Tri-Carb ${ }^{\circledR}$ 2000CA Liquid Scintillation Analyzer device; Packard, Meriden, CT). The percentage of complexes associated with the cells (in terms of DNA) was determined using the equation indicated above.

\section{Results}

Mean Diameter of Tf-Complexes

It is well recognized that the physicochemical properties of cationic liposome-DNA complexes strongly influence their 
biological activity (da Cruz et al., 2001; Eastman et al., 1997; Harvie et al., 2000). It has been largely reported that the size of the complexes affects their biodistribution profile upon administration in the blood circulation, extent of cell association and intracellular trafficking after internalization, resulting in different patterns of transfection (Lawrie et al., 1999; Tranchant et al., 2004; Wattiaux et al., 2000).

Figure 1 illustrates the influence of the ionic strength of the medium, mode of preparation and charge ratio of Tf-complexes on their final mean diameter. The final sizes of Tf-complexes are strongly affected by the ionic strength of the medium used in their preparation. As observed, the preparation of Tf-complexes in high-ionic strength medium (Fig. 1b) leads to the formation of 10 times larger particles than those prepared in low-ionic strength medium (Fig. 1a) since under these latter conditions particle aggregation is avoided. The order of addition of the components used in Tf-complex preparation also affects their final sizes. As illustrated in Figure 1, Tf-complexes prepared by order 2 exhibit slightly larger sizes compared to those prepared by order 1 . This effect is more evident for the $3 / 2(+/-)$ charge ratio, at which the complexes are essentially neutral independently of the ionic strength of the medium. This indicates that addition of Tf to the formed lipoplexes results in a more extensive aggregation of the complexes, leading to larger sizes.

\section{Protection of DNA by Tf-Complexes}

The capacity of the complexes to protect the carried genetic material against DNase degradation is one of the most important biophysical properties of the gene carriers. In fact, DNA has to be intact when it reaches the target cell for gene expression to occur; therefore, the carried gene material must be protected to avoid its degradation by the DNases present in the blood circulation and/or in the cell cytoplasm (Birchall, Kellaway \& Mills, 1999; da Cruz et al., 2001). The ability of Tf-complexes, prepared under the different experimental conditions, to protect the carried DNA was tested using the fluorescent probe PicoGreen, which exhibits high fluorescence intensity when it binds to double-stranded DNA. This assay is thus based on the decrease of fluorescence resulting from the decrease of PicoGreen accessibility to DNA upon complexation with the cationic liposomes (Fig. 2). In general, DexTf-complexes present a lower capacity to protect DNA (Fig. 2a) compared to HBS-Tf-complexes (Fig. 2b). As expected, the increase in the amount of cationic liposomes which results in higher Tf-complex charge ratios led to a higher degree of DNA protection, independently of the ionic strength of the medium used in their preparation. However, the results clearly show that the increase of DNA
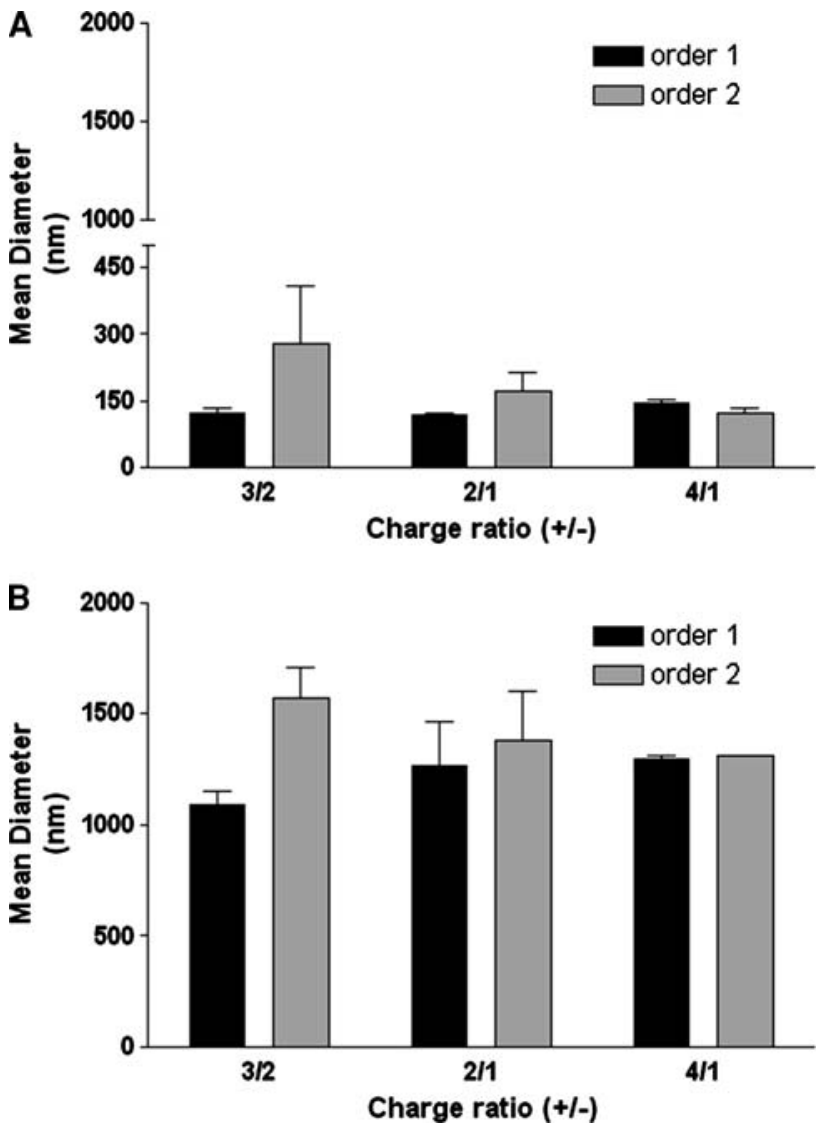

Fig. 1 Effect of lipid/DNA (+/-) charge ratio of Tf lipoplexes, mode of preparation and ionic strength of the medium on their mean diameter. Tf-complexes were prepared in (a) low-ionic strength medium (5\% Dex) and (b) high-ionic strength medium (HBS), in each case, either by adding Tf to the liposomes followed by addition of plasmid DNA (order 1) or by adding plasmid DNA to the liposomes followed by addition of $\mathrm{Tf}$ (order 2). Tf-complexes were prepared in a final volume of $1 \mathrm{ml}$ and diluted three times before size measurements in a Coulter N4 Plus instrument with angle fixed at $90^{\circ}$. All Tf-complexes were prepared immediately before analysis. Data represent the mean \pm SD of at least two independent experiments

protection with the increase of charge ratio was more pronounced for Dex-Tf-complexes than for HBSTf-complexes. The order of component addition influences the ability of Tf-complexes to protect DNA when they are prepared in Dex medium. When prepared by order 1, Dex-Tf-complexes exhibit a lower capacity to protect DNA than when order 2 was used in their preparation. Interestingly, a different behavior is observed for HBSTf-complexes, which exhibit similar capacity to protect DNA, independently of the order of component addition. Since for each type of complex (HBS- or Dex-Tf) the only difference in preparation is the order of addition of DNA and $\mathrm{Tf}$, these data suggest that the medium affects the mode of interaction of the components, namely, that of $\mathrm{Tf}$, which in turn modulates the capacity of the Tf-complexes to protect DNA. This differential behavior of 

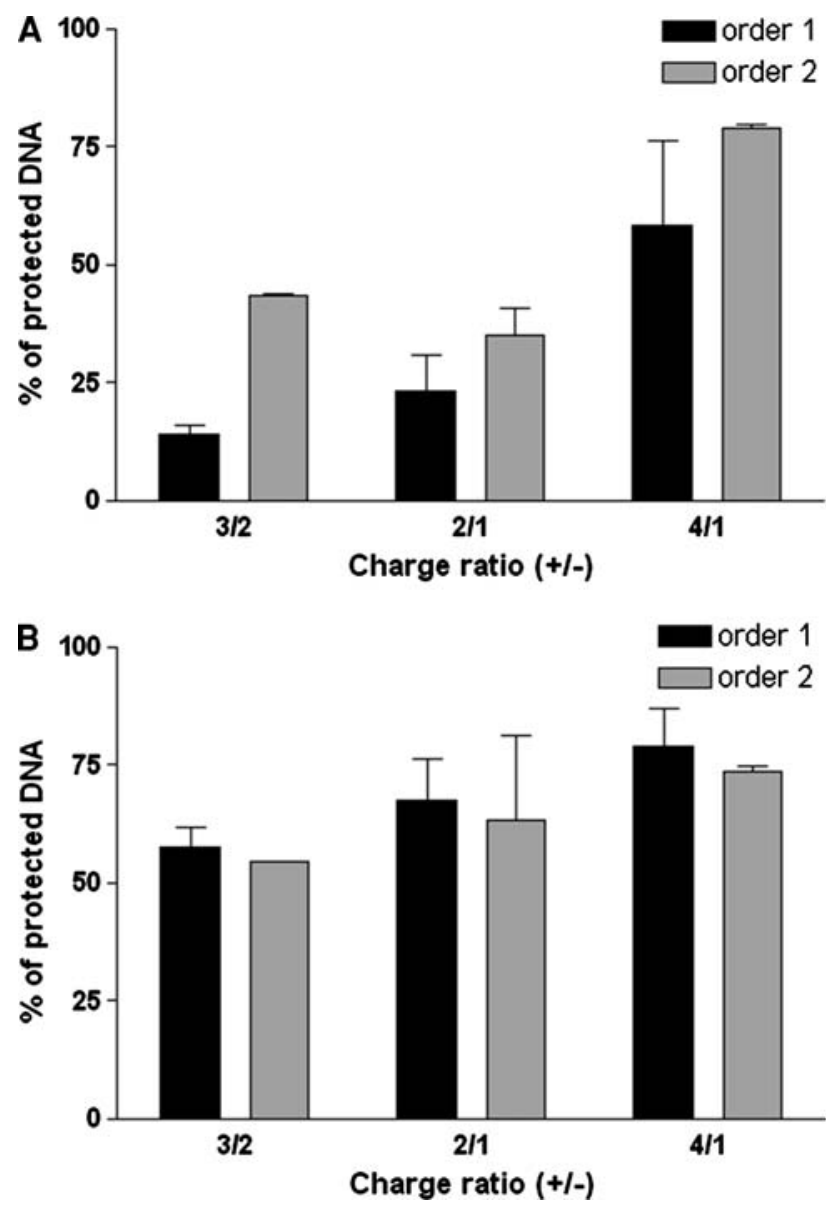

Fig. 2 Effect of lipid/DNA (+/-) charge ratio of Tf lipoplexes, mode of preparation and ionic strength of the medium on the protection of the associated DNA. Tf-complexes were prepared in (a) low-ionic strength medium (5\% Dex) and (b) high-ionic strength medium (HBS), in each case, by both orders 1 and 2. Tf-complexes containing $1 \mu \mathrm{g}$ of DNA were incubated with inactive or active DNase for $30 \mathrm{~min}$ at $37^{\circ} \mathrm{C}$, using 10 units of DNase $\mathrm{I} / \mu \mathrm{g}$ of DNA. The accessibility of PicoGreen to the DNA associated with the complexes was monitored for $2 \mathrm{~min}$ in a SPEX Fluorolog 2 fluorometer. The amount of DNA available to interact with the probe was calculated by subtracting the values of residual fluorescence (intrinsic fluorescence of the probe) from those obtained for the samples and expressed as the percentage of the control. Control corresponds to free DNA but in the same amount as that associated with the complexes (100\% PicoGreen accessibility). The results correspond to the mean \pm SD obtained from at least two independent experiments

Tf-complexes, prepared under the tested conditions, shows that the ionic strength of the medium has a determinant role in their ability to protect DNA.

\section{Biological Activity of Tf-Complexes}

In order to establish a possible dependence of the biological activity of Tf-complexes on their biophysical properties, transfection activity mediated by the Tfcomplexes, prepared under different experimental conditions, was evaluated.
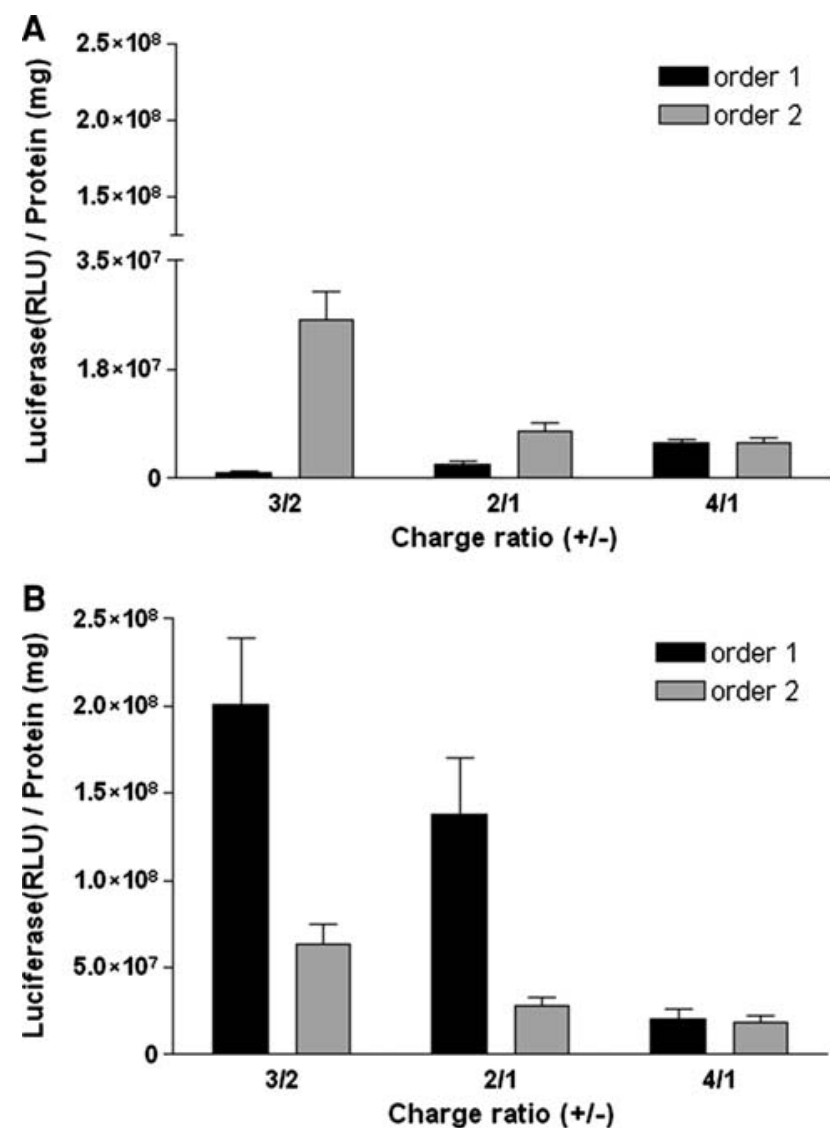

Fig. 3 Effect of lipid/DNA (+/-) charge ratio of Tf lipoplexes, mode of preparation and ionic strength of the medium on luciferase gene expression in COS-7 cells. Tf-complexes were prepared in (a) lowionic strength medium (5\% Dex) and (b) high-ionic strength medium (HBS), in each case, by both orders 1 and 2. After incubation of Tf-complexes containing $1 \mathrm{~g}$ of plasmid DNA with COS-7 cells for $4 \mathrm{~h}$, the medium was replaced with $1 \mathrm{ml}$ of medium containing $10 \%$ FBS and the cells were further incubated for $48 \mathrm{~h}$. The level of gene expression was evaluated as described in "Materials and Methods." The data are expressed as the percentage of the relative light units (RLU) of luciferase per milligram of total cell protein. The results correspond to the mean \pm SD obtained from triplicates and are representative of at least four independent experiments

Figure 3 illustrates the influence of the order of component addition, charge ratio and ionic strength of the medium used in Tf-complex preparation on their transfection activity in COS-7 cells. All the tested parameters influenced to some extent the ability of Tf-complexes to mediate gene delivery in vitro. The biological activity of HBS-Tf-complexes (Fig. 3b) is charge ratio-dependent, those prepared at a $3 / 2$ charge ratio being the most active. On the other hand, Dex-Tf-complexes (Fig. 3a) show a lower capacity to mediate transfection, the neutral complexes ( $3 / 2$ charge ratio) prepared by order 2 being the most efficient formulation.

As illustrated in Figure $3 \mathrm{~b}$, the order of component addition used in the preparation of HBS-Tf-complexes had a great influence on their biological activity. Although 
transfection activity decreases at charge ratios higher than $3 / 2$, HBS-Tf-complexes prepared by order 2 presented a lower capacity to transfect COS-7 cells than when prepared by order 1 , this difference being highly significant at $3 / 2$ and $2 / 1$ charge ratios.

It is interesting to note that the physicochemical properties of the Tf-complexes presented in Figures 1 and 2 did not correlate with their biological activity, particularly when the complexes were prepared in HBS. In fact, although the size and degree of DNA protection of HBSTf-complexes did not change appreciably for different lipid/DNA charge ratios and different orders of component addition, these parameters affected significantly their transfection capacity. A comparison between biological activity and ability to protect DNA allows us to conclude that Tf-complexes prepared by order 2 , although more efficient at protecting DNA at high charge ratios, are less efficient at mediating transfection than when prepared at low charge ratios.

In order to predict the in vivo behavior of Tf-complexes, their transfection activity was evaluated in the presence of serum. For this purpose, Tf-complexes prepared under different experimental conditions were added to COS-7 cells in medium containing different concentrations of serum. As observed in Table 1, although transfection activity of Dex-Tf-complexes did not change significantly with serum concentration, the effect of serum on transfection was highly dependent on the mode of Tf-complex preparation. In fact, while transfection mediated by Dex-Tf-complexes prepared at $3 / 2$ and $2 / 1$ charge ratios by order 1 remained essentially constant in the presence of serum (at the 4/1 charge ratio some variation could be detected), complexes prepared at these same charge ratios by order 2 showed a tendency to exhibit higher transfection activity in the presence of serum. On the other hand, our results show that the presence of serum had a great influence on the capacity of transfection of HBS-Tf-complexes, particularly at $20 \%$ serum concentration, under which conditions an enhancement of transfection was observed for all charges ratios tested. This effect was more pronounced for HBS-Tf-complexes with charge ratios close to neutral. It is possible that the type of interaction of these complexes with serum components resulted in the observed increase of transfection. Similar to what was observed for Dex-Tf-complexes, the mode of preparation of HBS-Tf-complexes affected their transfection activity, depending on whether this was assessed in the presence or absence of serum. In fact, while Tf-complexes prepared in HBS by order 1 were more efficient at mediating transfection in the presence of serum, transfection activity appeared to be insensitive to serum when order 2 was used in their preparation.

\section{Cell Association of Tf-Complexes}

In order to clarify the influence of the charge ratio, the mode of preparation and the ionic strength of the medium on transfection activity mediated by Tf lipoplexes, we examined the effect of these parameters on the extent of their association to COS-7 cells. Cell association encompasses the processes of binding, fusion with the plasma membrane, endocytosis and fusion with the endosomal membrane (da Cruz et al., 2001). For that purpose, Tf-complexes prepared under the different experimental conditions and containing rhodamine-labeled lipid or radioactive ${ }^{35} \mathrm{~S}$-labeled plasmid DNA were incubated with COS-7 cells at $37^{\circ} \mathrm{C}$, as described in "Materials and Methods."

Figure 4 shows that, in general, for all the studied conditions, HBS-Tf-complexes associated more extensively ( $\sim 1.8$ times) with COS-7 cells (Fig. $4 b$ ) than DexTf-complexes (Fig. 4a). In the case of Dex-Tf-complexes, no significant dependence of the extent of cell association on the

Table 1 Effect of serum on transfection mediated by Tf-complexes prepared in different experimental conditions

\begin{tabular}{|c|c|c|c|c|c|}
\hline \multirow[t]{2}{*}{ DOTAP:Chol/DNA } & \multirow[t]{2}{*}{$\%$ Serum } & \multicolumn{2}{|l|}{ Dex } & \multicolumn{2}{|l|}{ HBS } \\
\hline & & Order 1 & Order 2 & Order 1 & Order 2 \\
\hline \multirow[t]{3}{*}{$3 / 2$} & 0 & $1.38( \pm 0.59)$ & $38.58( \pm 9.49)$ & $302.65( \pm 84.83)$ & $96.28( \pm 23.00)$ \\
\hline & 20 & $0.19( \pm 0.26)$ & $26.41( \pm 35.40)$ & $981.38( \pm 381.41)$ & $92.56( \pm 35.25)$ \\
\hline & 40 & $2.00( \pm 2.26)$ & $83.71( \pm 99.55)$ & $319.41( \pm 56.13)$ & $28.93( \pm 9.06)$ \\
\hline \multirow[t]{3}{*}{$2 / 1$} & 0 & $2.97( \pm 2.69)$ & $10.80( \pm 4.61)$ & $195.59( \pm 124.55)$ & $35.90( \pm 20.04)$ \\
\hline & 20 & $0.87( \pm 1.71)$ & $30.61( \pm 76.03)$ & $1,304.91( \pm 530.75)$ & $150.44( \pm 68.62)$ \\
\hline & 40 & $0.18( \pm 0.16)$ & $16.57( \pm 8.93)$ & $492.44( \pm 124.10)$ & $63.26( \pm 19.30)$ \\
\hline \multirow[t]{3}{*}{$4 / 1$} & 0 & $5.74( \pm 1.61)$ & $6.60( \pm 3.56)$ & $16.61( \pm 3.50)$ & $25.46( \pm 9.39)$ \\
\hline & 20 & $8.45( \pm 2.58)$ & $5.30( \pm 7.21)$ & $496.31( \pm 95.83)$ & $193.28( \pm 125.34)$ \\
\hline & 40 & $2.45( \pm 2.35)$ & $6.33( \pm 4.12)$ & $195.48( \pm 31.76)$ & $46.48( \pm 19.43)$ \\
\hline
\end{tabular}

Tf-complexes were prepared as described in "Materials and Methods." The data, expressed as relative light units $\left(\times 10^{6}\right)$ per milligram of total cell protein, indicate the mean \pm SEM obtained from triplicates and are representative of three independent experiments 

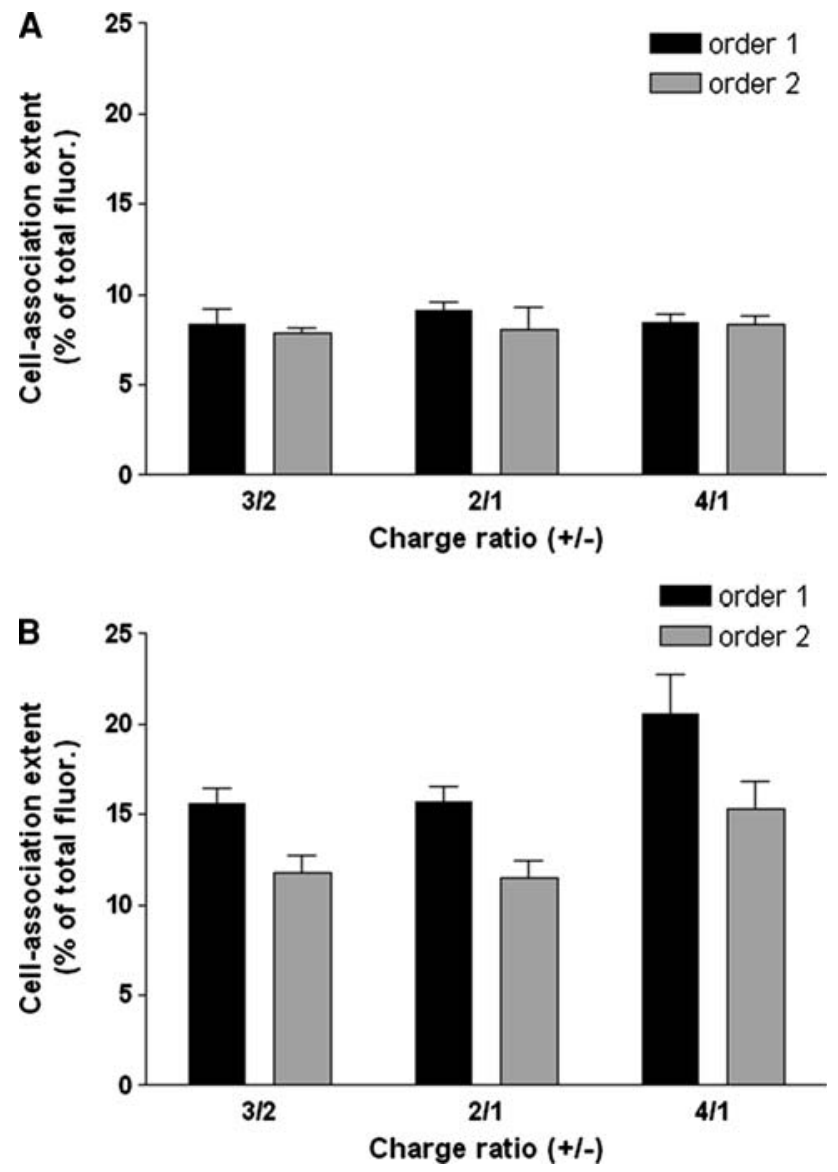

Fig. 4 Effect of lipid/DNA (+/-) charge ratio of Tf lipoplexes, mode of preparation and ionic strength of the medium on the extent of their association (in terms of lipid) to COS-7 cells. Tf-complexes were prepared in (a) low-ionic strength medium (5\% Dex) and (b) highionic strength medium (HBS), in each case, by both orders 1 and 2 . Liposomes containing DOTAP:Chol 1:1 were fluorescently labeled by incorporating rhodamine-PE into the lipid mixture at a concentration of $5 \mathrm{~mol} \%$ of total lipid. The extent of cell association was measured after 4-h incubation of the complexes with COS-7 cells at $37^{\circ} \mathrm{C}$, as described in "Materials and Methods." The data are expressed as a percentage of the total fluorescence and represent the mean \pm SD from two independent experiments carried out in triplicate

charge ratio or mode of preparation of the complexes was observed, while cell association of HBS-Tf-complexes depended on their charge ratio and mode of preparation. In fact, for each charge ratio tested, HBS-Tf-complexes prepared by order 1 associated more extensively with COS-7 cells than when prepared by order 2 . In order to complement these studies, parallel experiments on the extent of cell association of Tf-complexes were performed in terms of DNA included in the Tf-complexes. Results from these studies show that HBS-Tf-complexes present approximately 2.4 times more capacity to associate with $\mathrm{COS}-7$ cells (Fig. 5b) than Dex-Tf-complexes (Fig. 5a). In contrast to what was found for the extent of lipid-cell association, the extent of association of DNA carried by Dex-Tf-complexes
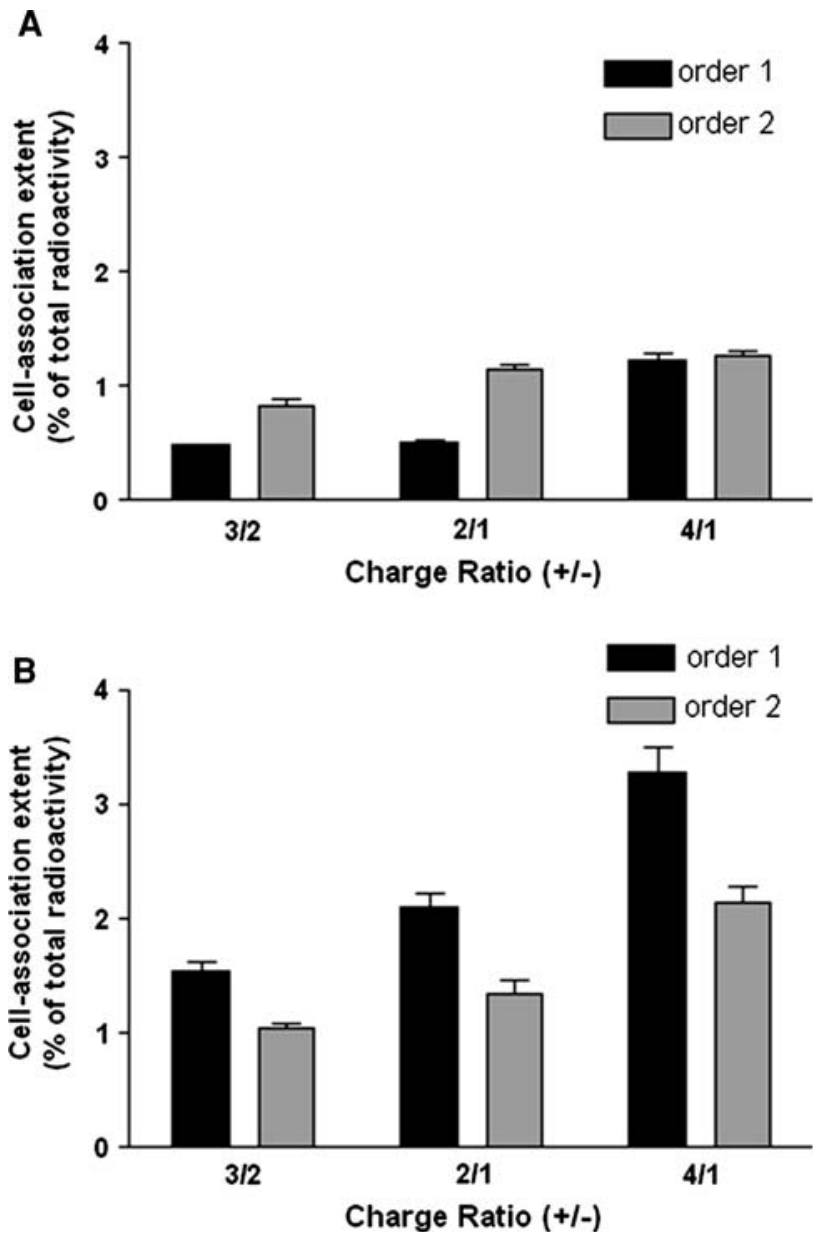

Fig. 5 Effect of lipid/DNA (+/-) charge ratio, mode of preparation and ionic strength of the medium on the extent of their association (in terms of DNA) to COS-7 cells. Tf-complexes were prepared in (a) low-ionic strength medium (5\% Dex) and (b) high-ionic strength medium (HBS), in each case, by both orders 1 and 2. Nucleotides labeled with radioactive ${ }^{35} \mathrm{~S}$ were diluted in the pDNA solution before complexation. The extent of cell association was measured after 4-h incubation with COS-7 cells at $37^{\circ} \mathrm{C}$, as described in "Materials and Methods." The data are expressed as a percentage of the total fluorescence and represent the mean \pm SD from two independent experiments carried out in triplicate

with COS-7 cells depended on the charge ratio and mode of preparation of these complexes. On the other hand, for the HBS-Tf-complexes the same dependence of cell association, in terms of DNA, as that evaluated in terms of lipid was observed. HBS-Tf-complexes prepared by order 1 presented an increased capacity to associate with the cells, which is in agreement with what was observed for lipid-cell association in these complexes.

As expected from the transfection studies, both lipid and DNA components of the HBS-Tf-complexes presented an increased capacity to associate with COS-7 cells compared to Dex-Tf-complexes. However, while HBS-Tf-complexes were approximately 200 times more efficient at mediating transfection than Dex-Tf-complexes, the extent of 
association of the carried DNA with the cells was only 2.4 times higher for the HBS-Tf-complexes than for the Dex-Tf-complexes, and the percentage of lipid-cell association was only 1.8 times larger for the former than for the latter. This finding clearly shows that besides binding and endocytosis, other steps play an important role in dictating the higher efficiency of transfection mediated by HBS-Tfcomplexes compared to Dex-Tf-complexes.

\section{Discussion}

One of the main goals in the field of gene transfer technology based on the use of DNA-condensing agents has been to set up a correlation between the biophysical properties of the resulting DNA complexes and their capacity to mediate transfection. The present study was aimed at understanding the effect of various factors involved in the preparation of Tf-complexes on their final physicochemical features and biological activity, which would facilitate the design of new vectors capable of fulfilling adequate properties, thus allowing their access to target cells and ensuring efficient gene expression.

Association of $\mathrm{Tf}$ to lipoplexes constitutes a promising strategy to enhance their capacity to mediate transfection (Cheng, 1996; da Cruz et al., 2001). In this work, Tf-complexes containing DOTAP/Chol were prepared at different lipid/DNA charge ratios under different experimental conditions, including the order of component addition and nature of the medium used in their preparation, and characterized in terms of physicochemical properties and capacity to mediate transfection in cultured cells, in both the presence and the absence of serum. Our studies were focused on COS-7 cells, which, being easily transfectable, would facilitate the analysis of the data. All the tested parameters, e.g., mode of preparation, ionic strength of the medium and charge ratio of Tf-complexes, influenced their capacity to mediate transfection. Tf-complexes prepared in HBS (HBS-Tf-complexes), by order 1 (cationic liposomes $+\mathrm{Tf}+\mathrm{pDNA}$ ) at charge ratios close to neutral presented the highest transfection efficacies (Fig. 3). Therefore, the preparation of Tf-complexes in HBS medium (high ionic strength) offers an advantage in terms of transfection compared to complexes prepared in 5\% Dex medium (low ionic strength), suggesting that the resulting complexes should exhibit different properties. A clear difference between the two types of complexes is their capacity to protect the carried genetic material. In general, HBS-Tf-complexes present a higher capacity to protect DNA than Dex-Tf-complexes (Fig. 2). Interestingly, when prepared by order 2 , the latter complexes presented a higher degree of DNA protection than when prepared by order 1 at all charge ratios tested. It is possible that Tf competes with DNA by interacting electrostatically with the positively charged liposomes (da Cruz et al., 2001). Thus, when the complexes are prepared by order 1 , less cationic charge will be available to complex with the added DNA than when order 2 is used in their preparation, thereby resulting in a lower DNA protection. This effect was observed for both types of complexes, irrespective of whether they were prepared in HBS or 5\% Dex. Such an effect was, however, more pronounced for Dex-Tf-complexes, which may be partly due to the influence of the ionic strength of the medium of complex preparation on the electrostatic interactions between charged counterions. Hirsch-Lerner and colleagues (2005) reported a reduction of DNA interaction with the cationic lipid when $\mathrm{NaCl}$ was present in the medium, which supports the observed differential behavior of Tf-complexes as depending on the ionic strength of the medium. A similar effect was observed for the size of the complexes, HBS-Tf-complexes being larger than Dex-Tf-complexes. Moreover, complexes prepared by order 2 tend to be larger than their congeners prepared by order 1 , showing that the order of Tf addition influences the final size of the complexes. In high-ionic strength solution the free counterions, like $\mathrm{Na}^{+}$or $\mathrm{Cl}^{-}$, can interact with charged regions of the particles present in solution, leading to a diminished electrostatic interaction between the charged particles, thus allowing other driving forces, like van der Waals interactions, to act and resulting in aggregation phenomena and formation of large but less compacted particles (da Cruz et al., 2001; Faneca, Simoes \& de Lima, 2002). When Tf-complexes are prepared in a low-ionic strength solution, because of the absence of counterions, the charged particles will interact avidly by electrostatic interactions, resulting in more compact and small particles due to electrostatic repulsions. Therefore, the ionic strength of the medium where the complexes are prepared influences the degree of interaction of the charged components, cationic liposomes and DNA, with a direct impact on DNA protection (Kennedy et al., 2000; Kichler et al., 1998; Scarzello et al., 2005; Zhang et al., 2003; Zuhorn et al., 2005). Moreover, Tf can also play a role in determining the final size of the complexes. Since the charge of $\mathrm{Tf}$ is not as localized as in cationic liposomes or in the DNA molecule, it is reasonable to assume that the effect of counterions present in solution will not be so pronounced. It is possible that in HBS medium Tf can act as an aggregation inducer, serving as a bridge between several complexes, thus leading to the increase in size of the resulting complexes, as can be observed in Figure $1 \mathrm{~b}$.

Interestingly, HBS-Tf-complexes prepared at low charge ratios by order 1 are the most efficient at mediating transfection (Fig. 3). The increased capacity of HBS-Tf-complexes to associate with the cells, in terms of both lipid and DNA, compared to Dex-Tf-complexes may 
partially justify that finding. Favorable interactions and binding to the cell surface as well as efficient protection of DNA against nucleases collectively concur with the increased transfection capacity exhibited by the HBS-Tfcomplexes (Konopka et al., 1996; Tomlinson \& Rolland, 1996; Zelphati et al., 1998). It has been clearly demonstrated that endocytosis constitutes the main route of cell entry of lipoplexes (Hui et al., 1996; Yu et al., 2004; Zabner et al., 1995; Zuhorn et al., 2002). Interestingly, Wattiaux et al. (2000) reported that complexes consisting of poly-L-lysine became trapped in the endosomes because their size impaired the transfer to lysosomes, which indicates the existence of a correlation between the size of the complexes and trafficking through the endocytic pathway. The authors concluded that reducing the rate of transfer of endocytosed DNA to lysosomes could promote transfection by allowing DNA to reside much longer in a prelysosomal compartment, sheltered from lysosomal nucleases, and therefore becoming available for transfer to the cytosol. Moreover, the size of the complexes per se can play a role in the escape from the endosome and influence intracellular trafficking to the nucleus (Escriou et al., 1998), thus having a direct effect on transfection levels. Indeed, large particles internalized into the cells lead to the formation of large intracellular vesicles that could be more easily destabilized, thus facilitating DNA escape (Xu \& Szoka, 1996). Taken together, these factors could explain why the large HBSTf-complexes are significantly more efficient at promoting transfection than Dex-Tf-complexes.

Our results on cell association (Figs. 4 and 5) show that HBS-Tf-complexes associate more extensively with COS-7 cells, in terms of both lipid and DNA, than Dex-Tf-complexes. As expected, the extent of cell association increases with the charge ratio of the complexes. However, the fact that transfection activity mediated by Tf-complexes decreases with increasing charge ratio, particularly for HBS-Tf-complexes, suggests that the extent of cell association is not a determinant factor for the transfection performance of the Tf-complexes. Three possible explanations for this finding are presented below:

1. Formation of inverted hexagonal structures: The capacity of the lipid incorporated in lipoplexes to form inverted hexagonal structures $\left(\mathrm{H}_{\mathrm{II}}\right)$ in the endosome has been reported to favor transfection by facilitating endosomal escape of DNA (Shi et al., 2002; Zuhorn et al., 2002, 2005). It has been reported that the ionic strength of the medium has a dramatic effect on the phase behavior of the lipids in the complexes (Scarzello et al., 2005; Shi et al., 2002; Smisterova et al., 2001). In this regard, the presence of $\mathrm{NaCl}$ in the medium may contribute to a reduction of the cross-sectional headgroup area, thus favoring the formation of $\mathrm{H}_{\mathrm{II}}$ phases.
2. Strength of DNA binding within the complex (effect of the order of component addition): The dissociation of DNA from the complexes and its escape from endosomes are crucial steps in the process of intracellular gene delivery and its consequent expression (Zabner et al., 1995). Therefore, it is reasonable to assume that any factor that influences any of these processes will have an effect on transfection activity. In the complexes prepared in HBS, the presence of counterions $\left(\mathrm{Na}^{+}\right.$and $\left.\mathrm{Cl}^{-}\right)$will weaken the electrostatic interaction between DNA and the cationic lipid. In fact, an extensive condensation of DNA may render the dissociation of the complex difficult, thus preventing intracellular delivery of DNA, which is in complete agreement with the observed transfection results. Accordingly, Tf-complexes prepared in HBS at charge ratios close to neutral, under which conditions DNA is believed not to be very condensed, are more efficient at mediating transfection compared to those prepared at higher charge ratios or in a low-ionic strength solution. The above explanation is strengthened by the increased ability of HBS-Tf-complexes to mediate transfection when prepared by order 1 , in which DNA will have to compete with $\mathrm{Tf}$, thus facilitating DNA release from the complex into the cell cytoplasm, which would then contribute to enhance transfection.

3. Role of transferrin in DNA escape from endosomes: Our previous results (Simoes et al., 1999) demonstrated that when associated with lipoplexes Tf promotes transfection, this enhancing effect being attributed to the following mechanisms: by triggering internalization of the complexes and by promoting cytoplasmic gene delivery in a $\mathrm{pH}$-dependent manner. Because HBS-Tf-complexes exhibit a larger size than Dex-Tf-complexes, they most likely have a higher number of associated Tf molecules, which will contribute to promote transfection. Besides size, the presence of a larger number of $\mathrm{Tf}$ molecules could explain why HBS-Tf-complexes associate more extensively with COS-7 cells, in terms of both lipid and DNA (Figs. 4 and 5). However, the role of Tf in promoting destabilization of the endosomes, by acting as a fusogenic protein, can be instrumental for the higher efficiency of HBS-Tf-complexes to mediate transfection compared to Dex-Tf-complexes (da Cruz et al., 2001).

Serum has been widely described as one of the major modulators of transfection mediated by cationic liposomes (Bally et al., 1999; Boukhnikachvili et al., 1997; Crook et al., 1998; Lawrie et al., 1999; Shi et al., 2002; Simoes et al., 2000), being largely reported as exhibiting an inhibitory effect on transfection (Harvie et al., 2000; 
Table 2 Effect of the experimental conditions used in the preparation of Tf-complexes on their physicochemical and biological properties

\begin{tabular}{llllll}
\hline & Size & DNA protection & Inhibitory effect of serum & Extent of cell association & Biological activity \\
\hline Ionic strength & +++ & ++ & $+/-$ & + & ++ \\
Charge ratio & + & ++ & +++ & + & +++ \\
Order of addition of the components & + & + & +++ & $++^{\mathrm{a}}$ & +++ \\
\hline
\end{tabular}

+++ , very high; ++, high; +, moderate; +/-, not relevant

${ }^{a}$ When complexes were prepared in high-ionic strength medium

Lawrie et al., 1999; Nchinda, Uberla \& Zschornig, 2002; Shi et al., 2002). Transfection studies performed in vitro in the presence of serum aim at emulating in vivo conditions (Koynova \& MacDonald, 2005; Nchinda et al., 2002; Simberg, Weiss \& Barenholz, 2005; Vitiello et al., 1998). The results presented in Table 1 clearly show that the modulating effect of serum on transfection mediated by Tfcomplexes is dependent on all the studied parameters. Transfection mediated by HBS-Tf-complexes in the presence of serum was greatly potentiated at all charges ratios tested, particularly for those prepared at charge ratios close to neutral, independently of the order of component addition. Albumin has been shown to enhance transfection mediated by cationic liposomes, which was explained by its ability to undergo a low pH-induced conformational change, thereby acquiring fusogenic properties (Simoes et al., 2000; Garcia et al., 1983; Schenkman et al., 1981), similarly to $\mathrm{Tf}$. It is possible that Tf-complexes that are more prone to interact with albumin present in the medium, while maintaining their capacity to enter the cells, could gain advantage over Tf-complexes that do not present this capacity during the transfection process. In this context, it is possible that HBS-Tf-complexes prepared at charge ratios close to neutral, because of their instability, could interact easily with albumin present in the medium, which could explain the high efficacies of transfection observed in the presence of $20 \%$ serum. Moreover, it is interesting to note that HBS-Tf-complexes prepared by order 1 showed a transfection capacity several times higher than that achieved when order 2 was used. Under the latter conditions, Tf may be located on the surface of the complexes, thus hindering their interaction with albumin. On the other hand, HBS-Tf-complexes prepared by order 1 could be more available to interact with albumin, thus explaining their improved transfection efficiency in the presence of serum. However, the fact that Dex-Tf-complexes present a low tendency to interact with serum components, associated with their reduced size, can constitute an important advantage to their use in vivo, namely, if the intravenous route is chosen. For instance, the reduced size of Dex-Tf-complexes can be advantageous to passive targeting to tumor cells, where the vascular endothelium is relatively leaky and therefore small particles can extravasate and accumulate in the tumor (Torchilin, 2000). Table 2 summarizes the main findings of the present work, which clearly show that the physicochemical properties of DNA carriers based on cationic liposomes depend on the ionic strength of the medium used in their preparation, order of component addition and lipid/ DNA charge ratio. We conclude that the complexes that are both most active and efficient at protecting the carried DNA are those prepared in high-ionic strength medium and at charge ratios close to neutral. The observation of enhanced transfection mediated by HBS-Tf-complexes in the presence of serum is particularly relevant, suggesting that these complexes deserve to be explored for in vivo use.

Overall, the results from these studies may help in the future design of more efficient cationic lipid-based particles, namely for in vivo applications.

Acknowledgements N. P. is the recipient of a fellowship from the Portuguese Foundation for Science and Technology (SFRH/BD/6135/ 2001). This work was partially financed by a grant from the Portuguese Foundation for Science and Technology (PTDC/BIO/65627/2006).

\section{References}

Baekelandt V, De Strooper B, Nuttin B, Debyser Z (2000) Gene therapeutic strategies for neurodegenerative diseases. Curr Opin Mol Ther 2:540-554

Bally MB, Harvie P, Wong FM, Kong S, Wasan EK, Reimer DL (1999) Biological barriers to cellular delivery of lipid-based DNA carriers. Adv Drug Deliv Rev 38:291-315

Birchall JC, Kellaway IW, Mills SN (1999) Physico-chemical characterisation and transfection efficiency of lipid-based gene delivery complexes. Int J Pharm 183:195-207

Boukhnikachvili T, Aguerre-Chariol O, Airiau M, Lesieur S, Ollivon M, Vacus J (1997) Structure of in-serum transfecting DNAcationic lipid complexes. FEBS Lett 409:188-194

Cheng PW (1996) Receptor ligand-facilitated gene transfer: enhancement of liposome-mediated gene transfer and expression by transferrin. Hum Gene Ther 7:275-282

Crook K, Stevenson BJ, Dubouchet M, Porteous DJ (1998) Inclusion of cholesterol in DOTAP transfection complexes increases the delivery of DNA to cells in vitro in the presence of serum. Gene Ther 5:137-143

da Cruz MT, Simoes S, Pires PP, Nir S, de Lima MC (2001) Kinetic analysis of the initial steps involved in lipoplex-cell interactions: effect of various factors that influence transfection activity. Biochim Biophys Acta 1510:136-151 
da Cruz MT, Simoes S, de Lima MC (2004) Improving lipoplexmediated gene transfer into C6 glioma cells and primary neurons. Exp Neurol 187:65-75

da Cruz MT, Cardoso AL, de Almeida LP, Simoes S, de Lima MC (2005) Tf-lipoplex-mediated NGF gene transfer to the CNS: neuronal protection and recovery in an excitotoxic model of brain injury. Gene Ther 12:1242-1252

Dropulic B, June CH (2006) Gene-based immunotherapy for human immunodeficiency virus infection and acquired immunodeficiency syndrome. Hum Gene Ther 17:577-588

Eastman SJ, Siegel C, Tousignant J, Smith AE, Cheng SH, Scheule RK (1997) Biophysical characterization of cationic lipid: DNA complexes. Biochim Biophys Acta 1325:41-62

Escriou V, Ciolina C, Lacroix F, Byk G, Scherman D, Wils P (1998) Cationic lipid-mediated gene transfer: effect of serum on cellular uptake and intracellular fate of lipopolyamine/DNA complexes. Biochim Biophys Acta 1368:276-288

Faneca H, Simoes S, de Lima MC (2002) Evaluation of lipid-based reagents to mediate intracellular gene delivery. Biochim Biophys Acta 1567:23-33

Galanis E, Russell S (2001) Cancer gene therapy clinical trials: lessons for the future. Br J Cancer 85:1432-1436

Garcia LA, Schenkman S, Araujo PS, Chaimovich H (1983) Fusion of small unilamellar vesicles induced by bovine serum albumin fragments. Braz J Med Biol Res 16:89-96

Harvie P, Wong FM, Bally MB (2000) Use of poly(ethylene glycol)lipid conjugates to regulate the surface attributes and transfection activity of lipid-DNA particles. J Pharm Sci 89:652-663

Hirsch-Lerner D, Zhang M, Eliyahu H, Ferrari ME, Wheeler CJ, Barenholz Y (2005) Effect of "helper lipid" on lipoplex electrostatics. Biochim Biophys Acta 1714:71-84

Horner AA, Van Uden JH, Zubeldia JM, Broide D, Raz E (2001) DNA-based immunotherapeutics for the treatment of allergic disease. Immunol Rev 179:102-118

Huckett B, Ariatti M, Hawtrey AO (1990) Evidence for targeted gene transfer by receptor-mediated endocytosis. Stable expression following insulin-directed entry of NEO into HepG2 cells. Biochem Pharmacol 40:253-263

Hui SW, Langner M, Zhao YL, Ross P, Hurley E, Chan K (1996) The role of helper lipids in cationic liposome-mediated gene transfer. Biophys J 71:590-599

Kennedy MT, Pozharski EV, Rakhmanova VA, MacDonald RC (2000) Factors governing the assembly of cationic phospholipids-DNA complexes. Biophys J 78:1620-1633

Kichler A, Zauner W, Ogris M, Wagner E (1998) Influence of the DNA complexation medium on the transfection efficiency of lipospermine/DNA particles. Gene Ther 5:855-860

Kikuchi A, Sugaya S, Ueda H, Tanaka K, Aramaki Y, Hara T, Arima H, Tsuchiya S, Fuwa T (1996) Efficient gene transfer to EGF receptor overexpressing cancer cells by means of EGF-labeled cationic liposomes. Biochem Biophys Res Commun 227:666671

Konopka K, Pretzer E, Felgner PL, Duzgunes N (1996) Human immunodeficiency virus type-1 (HIV-1) infection increases the sensitivity of macrophages and THP- 1 cells to cytotoxicity by cationic liposomes. Biochim Biophys Acta 1312:186-196

Koynova R, MacDonald RC (2005) Lipid transfer between cationic vesicles and lipid-DNA lipoplexes: effect of serum. Biochim Biophys Acta 1714:63-70

Lawrie A, Brisken AF, Francis SE, Tayler DI, Chamberlain J, Crossman DC, Cumberland DC, Newman CM (1999) Ultrasound enhances reporter gene expression after transfection of vascular cells in vitro. Circulation 99:2617-2620

Lee RJ, Huang L (1996) Folate-targeted, anionic liposome-entrapped polylysine-condensed DNA for tumor cell-specific gene transfer. J Biol Chem 271:8481-8487
Lewis JG, Lin KY, Kothavale A, Flanagan WM, Matteucci MD, DePrince RB, Mook RA Jr, Hendren RW, Wagner RW (1996) A serum-resistant cytofectin for cellular delivery of antisense oligodeoxynucleotides and plasmid DNA. Proc Natl Acad Sci USA 93:3176-3181

Liu Y, Mounkes LC, Liggitt HD, Brown CS, Solodin I, Heath TD, Debs RJ (1997) Factors influencing the efficiency of cationic liposome-mediated intravenous gene delivery. Nat Biotechnol 15:167-173

Mahato RI, Anwer K, Tagliaferri F, Meaney C, Leonard P, Wadhwa MS, Logan M, French M, Rolland A (1998) Biodistribution and gene expression of lipid/plasmid complexes after systemic administration. Hum Gene Ther 9:2083-2099

Mulligan RC (1993) The basic science of gene therapy. Science 260:926-932

Nchinda G, Uberla K, Zschornig O (2002) Characterization of cationic lipid DNA transfection complexes differing in susceptibility to serum inhibition. BMC Biotechnol 2:12

Neves SS, Sarmento-Ribeiro AB, Simoes S, Pedroso de Lima MC (2006) Transfection of oral cancer cells mediated by transferrinassociated lipoplexes: mechanisms of cell death induced by herpes simplex virus thymidine kinase/ganciclovir therapy. Biochim Biophys Acta 1758:1703-1712

Scarzello M, Chupin V, Wagenaar A, Stuart MC, Engberts JB, Hulst $R$ (2005) Polymorphism of pyridinium amphiphiles for gene delivery: influence of ionic strength, helper lipid content, and plasmid DNA complexation. Biophys J 88:2104-2113

Schenkman S, Araujo PS, Dijkman R, Quina FH, Chaimovich H (1981) Effects of temperature and lipid composition on the serum albumin-induced aggregation and fusion of small unilamellar vesicles. Biochim Biophys Acta 649:633-647

Sedmak JJ, Grossberg SE (1977) A rapid, sensitive, and versatile assay for protein using Coomassie brilliant blue G250. Anal Biochem 79:544-552

Shi F, Wasungu L, Nomden A, Stuart MC, Polushkin E, Engberts JB, Hoekstra D (2002) Interference of poly(ethylene glycol)-lipid analogues with cationic-lipid-mediated delivery of oligonucleotides; role of lipid exchangeability and non-lamellar transitions. Biochem J 366:333-341

Shirasaka Y, Ito M, Mitsuyoshi I, Mikawa H, Serikawa T, Yamada J (1991) Benzodiazepine receptor binding in the spontaneously epileptic rat and its parent strains. Exp Neurol 113:255-259

Simberg D, Weiss A, Barenholz Y (2005) Reversible mode of binding of serum proteins to DOTAP/cholesterol lipoplexes: a possible explanation for intravenous lipofection efficiency. Hum Gene Ther 16:1087-1096

Simoes S, Slepushkin V, Pires P, Gaspar R, de Lima MP, Duzgunes N (1999) Mechanisms of gene transfer mediated by lipoplexes associated with targeting ligands or $\mathrm{pH}$-sensitive peptides. Gene Ther 6:1798-1807

Simoes S, Slepushkin V, Pires P, Gaspar R, Pedroso de Lima MC, Duzgunes N (2000) Human serum albumin enhances DNA transfection by lipoplexes and confers resistance to inhibition by serum. Biochim Biophys Acta 1463:459-469

Smisterova J, Wagenaar A, Stuart MC, Polushkin E, ten Brinke G, Hulst R, Engberts JB, Hoekstra D (2001) Molecular shape of the cationic lipid controls the structure of cationic lipid/dioleylphosphatidylethanolamine-DNA complexes and the efficiency of gene delivery. J Biol Chem 276:47615-47622

Solodin I, Brown CS, Bruno MS, Chow CY, Jang EH, Debs RJ, Heath TD (1995) A novel series of amphiphilic imidazolinium compounds for in vitro and in vivo gene delivery. Biochemistry 34:13537-13544

Tomlinson E, Rolland AP (1996) Controllable gene therapy pharmaceutics of nonviral gene delivery systems. J Control Release $39: 357-372$ 
Torchilin VP (2000) Drug targeting. Eur J Pharm Sci 11(Suppl 2):S81-S91

Tranchant I, Thompson B, Nicolazzi C, Mignet N, Scherman D (2004) Physicochemical optimisation of plasmid delivery by cationic lipids. J Gene Med 6(Suppl 1):S24-S35

Vitiello L, Bockhold K, Joshi PB, Worton RG (1998) Transfection of cultured myoblasts in high serum concentration with DODAC:DOPE liposomes. Gene Ther 5:1306-1313

Wattiaux R, Laurent N, Wattiaux-De Coninck S, Jadot M (2000) Endosomes, lysosomes: their implication in gene transfer. Adv Drug Deliv Rev 41:201-208

Wheeler CJ, Felgner PL, Tsai YJ, Marshall J, Sukhu L, Doh SG, Hartikka J, Nietupski J, Manthorpe M, Nichols M, Plewe M, Liang X, Norman J, Smith A, Cheng SH (1996) A novel cationic lipid greatly enhances plasmid DNA delivery and expression in mouse lung. Proc Natl Acad Sci USA 93:11454-11459

Xu Y, Szoka FC Jr (1996) Mechanism of DNA release from cationic liposome/DNA complexes used in cell transfection. Biochemistry 35:5616-5623

Yu W, Pirollo KF, Rait A, Yu B, Xiang LM, Huang WQ, Zhou Q, Ertem G, Chang EH (2004) A sterically stabilized immunolipoplex for systemic administration of a therapeutic gene. Gene Ther 11:1434-1440

Zabner J, Fasbender AJ, Moninger T, Poellinger KA, Welsh MJ (1995) Cellular and molecular barriers to gene transfer by a cationic lipid. J Biol Chem 270:18997-19007

Zelphati O, Nguyen C, Ferrari M, Felgner J, Tsai Y, Felgner PL (1998) Stable and monodisperse lipoplex formulations for gene delivery. Gene Ther 5:1272-1282

Zhang Y, Garzon-Rodriguez W, Manning MC, Anchordoquy TJ (2003) The use of fluorescence resonance energy transfer to monitor dynamic changes of lipid-DNA interactions during lipoplex formation. Biochim Biophys Acta 1614:182-192

Zuhorn IS, Oberle V, Visser WH, Engberts JB, Bakowsky U, Polushkin E, Hoekstra D (2002) Phase behavior of cationic amphiphiles and their mixtures with helper lipid influences lipoplex shape, DNA translocation, and transfection efficiency. Biophys J 83:2096-2108

Zuhorn IS, Bakowsky U, Polushkin E, Visser WH, Stuart MC, Engberts JB, Hoekstra D (2005) Nonbilayer phase of lipoplexmembrane mixture determines endosomal escape of genetic cargo and transfection efficiency. Mol Ther 11:801-810 